\title{
The new UP-isomorphism theorems for UP-algebras in the meaning of the congruence determined by a UP-homomorphism
}

\author{
Phakawat Mosrijai $^{\mathrm{a}}$, Akarachai Satirad ${ }^{\mathrm{a}}$ and Aiyared Iampan $^{\mathrm{a}^{*}}$ \\ ${ }^{a}$ Department of Mathematics, School of Science, University of Phayao, Phayao 56000, Thailand \\ *Corresponding author E-mail: aiyared.ia@up.ac.th
}

Article Info
Keywords: UP-algebra, UP-
homomorphism, Fundamental
theorem, UP-isomorphism theorem.
2010 AMS: 03 G25, $06 F 35$
Received: 16 March 2018

Accepted: 19 May 2018

Available online: 30 June 2018

\begin{abstract}
The aim of this paper is to construct the new fundamental theorem of UP-algebras in the meaning of the congruence determined by a UP-homomorphism. We also give an application of the theorem to the first, second, and third UP-isomorphism theorems in UP-algebras.
\end{abstract}

\section{Introduction and preliminaries}

Among many algebraic structures, algebras of logic form important class of algebras. Examples of these are BCK-algebras [7], BCI-algebras [8], BCH-algebras [4], KU-algebras [15], SU-algebras [10], UP-algebras [6] and others. They are strongly connected with logic. For example, BCI-algebras introduced by Iséki [8] in 1966 have connections with BCI-logic being the BCI-system in combinatory logic which has application in the language of functional programming. BCK and BCI-algebras are two classes of logical algebras. They were introduced by Imai and Iséki [7, 8] in 1966 and have been extensively investigated by many researchers. It is known that the class of BCK-algebras is a proper subclass of the class of BCI-algebras.

The isomorphism theorems play an important role in a general logical algebra, which were studied by several researches such as: In 1998, Jun, Hong, Xin and Roh [9] proved isomorphism theorems by using Chinese Remainder Theorem in BCI-algebras. In 2001, Park, Shim and Roh [14] proved isomorphism theorems of IS-algebras. In 2004, Hao and Li [3] introduced the concept of ideals of an ideal in a BCI-algebra and some isomorphism theorems are obtained by using this concept. They obtained several isomorphism theorems of BG-algebras and related properties. In 2006, Kim [12] introduced the notion of KS-semigroups. He characterized ideals of a KS-semigroup and proved the first isomorphism theorem for KS-semigroups. In 2007, Dar and Akram [2] introduced the notion of K-homomorphism of K-algebras. In 2008, Kim and Kim [11] introduced the notion of BG-algebras which is a generalization of B-algebras. They obtained several isomorphism theorems of BG-algebras and related properties. In 2009, Paradero-Vilela and Cawi [13] characterized KS-semigroup homomorphisms and proved the isomorphism theorems for KS-semigroups. In 2011, Keawrahun and Leerawat [10] introduced the notion of SU-semigroups and proved the isomorphism theorems for SU-semigroups. In 2012, Asawasamrit [1] introduced the notion of KK-algebras and studied isomorphism theorems of KK-algebras. In 2015, Iampan [5] studied UP-isomorphism theorems of UP-algebras.

In this paper, we construct the new fundamental theorem of UP-algebras in the meaning of the congruence determined by a UP-homomorphism. We also give an application of the theorem to the first, second, and third UP-isomorphism theorems in UP-algebras.

Before we begin our study, we will introduce to the definition of a UP-algebra.

Definition 1.1. [6] An algebra $A=(A, \cdot, 0)$ of type $(2,0)$ is called a UP-algebra, where A is a nonempty set, $\cdot$ is a binary operation on $A$, and 0 is a fixed element of $A$ (i.e., a nullary operation) if it satisfies the following axioms: for any $x, y, z \in A$,

(UP-1) $(y \cdot z) \cdot((x \cdot y) \cdot(x \cdot z))=0$,

(UP-2) $0 \cdot x=x$,

(UP-3) $x \cdot 0=0$,

(UP-4) $x \cdot y=y \cdot x=0$ implies $x=y$. 
Example 1.2. [6] Let $X$ be a universal set. Define two binary operations $\cdot$ and $*$ on the power set of $X$ by putting $A \cdot B=B \cap A^{\prime}$ and $A * B=B \cup A^{\prime}$ for all $A, B \in \mathscr{P}(X)$. Then $(\mathscr{P}(X), \cdot, \emptyset)$ and $(\mathscr{P}(X), *, X)$ are UP-algebras and we shall call it the power UP-algebra of type 1 and the power UP-algebra of type 2, respectively.

Example 1.3. [6] Let $A=\{0, a, b, c\}$ be a set with a binary operation · defined by the following Cayley table:

\begin{tabular}{c|cccc}
$\cdot$ & 0 & $a$ & $b$ & $c$ \\
\hline 0 & 0 & $a$ & $b$ & $c$ \\
$a$ & 0 & 0 & 0 & 0 \\
$b$ & 0 & $a$ & 0 & $c$ \\
$c$ & 0 & $a$ & $b$ & 0
\end{tabular}

Then $(A, \cdot, 0)$ is a UP-algebra.

In what follows, let $A$ and $B$ denote UP-algebras unless otherwise specified. The following proposition is very important for the study of UP-algebras.

Proposition 1.4. [6] In a UP-algebra A, the following properties hold: for any $x, y, z \in A$,

(1) $x \cdot x=0$,

(2) $x \cdot y=0$ and $y \cdot z=0$ implies $x \cdot z=0$,

(3) $x \cdot y=0$ implies $(z \cdot x) \cdot(z \cdot y)=0$,

(4) $x \cdot y=0$ implies $(y \cdot z) \cdot(x \cdot z)=0$,

(5) $x \cdot(y \cdot x)=0$,

(6) $(y \cdot x) \cdot x=0$ if and only if $x=y \cdot x$, and

(7) $x \cdot(y \cdot y)=0$.

Definition 1.5. [6] Let A be a UP-algebra. A nonempty subset B of A is called a UP-ideal of A if it satisfies the following properties:

(1) the constant 0 of $A$ is in $B$, and

(2) for any $x, y, z \in A, x \cdot(y \cdot z) \in B$ and $y \in B$ implies $x \cdot z \in B$.

Definition 1.6. [6] Let $A=(A, \cdot, 0)$ be a UP-algebra. A subset $S$ of $A$ is called a UP-subalgebra of $A$ if the constant 0 of $A$ is in $S$, and $(S, \cdot, 0)$ itself forms a UP-algebra.

Proposition 1.7. [6] A nonempty subset $S$ of a UP-algebra $A=(A, \cdot, 0)$ is a UP-subalgebra of $A$ if and only if $S$ is closed under the . multiplication on $A$.

Definition 1.8. [6] Let A be a UP-algebra. An equivalence relation $\rho$ on $A$ is called a congruence if for any $x, y, z \in A$,

$$
x \rho y \text { implies } x \cdot z \rho y \cdot z \text { and } z \cdot x \rho z \cdot y \text {. }
$$

Lemma 1.9. [6] An equivalence relation $\rho$ on $A$ is a congruence if and only if for any $x, y, u, v \in A$, x $\rho y$ and $u \rho v$ imply $x \cdot u \rho y \cdot v$.

Definition 1.10. [6] Let $A$ be a UP-algebra and B a UP-ideal of A. Define the binary relation $\sim_{B}$ on $A$ as follows: for all $x, y \in A$,

$$
x \sim_{B} y \text { if and only if } x \cdot y \in B \text { and } y \cdot x \in B .
$$

Proposition 1.11. [6] Let A be a UP-algebra and B a UP-ideal of A with a binary relation $\sim_{B}$ defined by (1.2). Then $\sim_{B}$ is a congruence on $A$.

Let $A$ be a UP-algebra and $\rho$ a congruence on $A$. If $x \in A$, then the $\rho$-class of $x$ is the $(x) \rho$ defined as follows:

$$
(x)_{\rho}=\{y \in A \mid y \rho x\} .
$$

Then the set of all $\rho$-classes is called the quotient set of $A$ by $\rho$, and is denoted by $A / \rho$. That is,

$$
A / \rho=\left\{(x)_{\rho} \mid x \in A\right\}
$$

Theorem 1.12. [6] Let A be a UP-algebra and $B$ a UP-ideal of $A$. Then $\left(A / \sim_{B}, *,(0)_{\sim_{B}}\right)$ is a UP-algebra under the $*$ multiplication defined by $(x)_{\sim_{B}} *(y)_{\sim_{B}}=(x \cdot y)_{\sim_{B}}$ for all $x, y \in A$, called the quotient UP-algebra of $A$ induced by the congruence $\sim_{B}$.

Definition 1.13. [6] Let $(A, \cdot, 0)$ and $\left(A^{\prime}, \cdot^{\prime}, 0^{\prime}\right)$ be UP-algebras. A mapping from A to $A^{\prime}$ is called a UP-homomorphism if

$$
f(x \cdot y)=f(x) \cdot^{\prime} f(y) \text { for all } x, y \in A .
$$

A UP-homomorphism $f: A \rightarrow A^{\prime}$ is called a

(1) UP-epimorphism if $f$ is surjective,

(2) UP-monomorphism if $f$ is injective,

(3) UP-isomorphism if $f$ is bijective. Moreover, we say $A$ is UP-isomorphic to $A^{\prime}$, symbolically, $A \cong A^{\prime}$, if there is a UP-isomorphism from A to $A^{\prime}$. 
Let $f$ be a mapping from $A$ to $A^{\prime}$, and let $B$ be a nonempty subset of $A$, and $B^{\prime}$ of $A^{\prime}$. The set $\{f(x) \mid x \in B\}$ is called the image of $B$ under $f$, denoted by $f(B)$. In particular, $f(A)$ is called the image of $f$, denoted by $\operatorname{Im}(f)$. Dually, the set $\left\{x \in A \mid f(x) \in B^{\prime}\right\}$ is said the inverse image of $B^{\prime}$ under $f$, symbolically, $f^{-1}\left(B^{\prime}\right)$. Especially, we say $f^{-1}\left(\left\{0^{\prime}\right\}\right)$ is the kernel of $f$, written by $\operatorname{Ker}(f)$. That is,

$$
\operatorname{Im}(f)=\left\{f(x) \in A^{\prime} \mid x \in A\right\}
$$

and

$$
\operatorname{Ker}(f)=\left\{x \in A \mid f(x)=0^{\prime}\right\} .
$$

Theorem 1.14. [6] Let $A$ be a UP-algebra and B a UP-ideal of $A$. Then the mapping $\pi_{B}: A \rightarrow A / \sim_{B}$ defined by $\pi_{B}(x)=(x)_{\sim_{B}}$ for all $x \in A$ is a UP-epimorphism, called the natural projection from $A$ to $A / \sim_{B}$.

On a UP-algebra $A=(A, \cdot, 0)$, we define a binary relation $\leq$ on $A$ as follows: for all $x, y \in A$,

$$
x \leq y \text { if and only if } x \cdot y=0 .
$$

Proposition 1.15. [6] Let $A$ be a UP-algebra with a binary relation $\leq$ defined by (1.3). Then $(A, \leq)$ is a partially ordered set with 0 as the greatest element.

We often call the partial ordering $\leq$ defined by (1.3) the UP-ordering on $A$. From now on, the symbol $\leq$ will be used to denote the UP-ordering, unless specified otherwise.

Theorem 1.16. [6] Let $\left(A, \cdot, 0_{A}\right)$ and $\left(B, *, 0_{B}\right)$ be UP-algebras and let $f: A \rightarrow B$ be a UP-homomorphism. Then the following statements hold:

(1) $f\left(0_{A}\right)=0_{B}$,

(2) for any $x, y \in A$, if $x \leq y$, then $f(x) \leq f(y)$,

(3) if $C$ is a UP-subalgebra of $A$, then the image $f(C)$ is a UP-subalgebra of $B$. In particular, $\operatorname{Im}(f)$ is a UP-subalgebra of $B$,

(4) if $D$ is a UP-subalgebra of $B$, then the inverse image $f^{-1}(D)$ is a UP-subalgebra of $A$. In particular, $\operatorname{Ker}(f)$ is a UP-subalgebra of $A$,

(5) if $C$ is a UP-ideal of $A$ such that $\operatorname{Ker}(f) \subseteq C$, then the image $f(C)$ is a UP-ideal of $f(A)$,

(6) if $D$ is a UP-ideal of $B$, then the inverse image $f^{-1}(D)$ is a UP-ideal of $A$. In particular, $\operatorname{Ker}(f)$ is a UP-ideal of $A$, and

(7) $\operatorname{Ker}(f)=\left\{0_{A}\right\}$ if and only if $f$ is injective.

\section{Main results}

In this section, we introduce the congruence determined by a UP-homomorphism and prove the new fundamental theorem of UP-algebras in the meaning of the congruence determined by a UP-homomorphism. We also prove the first, second, and third UP-isomorphism theorems in UP-algebras.

Definition 2.1. Let $\left(A, \cdot, 0_{A}\right)$ and $\left(B, \bullet, 0_{B}\right)$ be UP-algebras, and $f: A \rightarrow B$ a UP-homomorphism. Define the binary relation $\sim_{f}$ on $A$ as follows: for all $x, y \in A$,

$$
x \sim_{f} y \text { if and only if } f(x)=f(y) .
$$

Theorem 2.2. Let $\left(A, \cdot, 0_{A}\right)$ and $\left(B, \bullet, 0_{B}\right)$ be UP-algebras, and $f: A \rightarrow B$ a UP-homomorphism with a binary relation $\sim_{f}$ on $A$ defined by (2.1). Then $\sim_{f}$ is a congruence on A, called the congruence determined by $f$.

Proof. Reflexive: For all $x \in A$, we have $f(x)=f(x)$. Thus $x \sim_{f} x$.

Symmetric: Let $x, y \in A$ be such that $x \sim_{f} y$. Then $f(x)=f(y)$, so $f(y)=f(x)$. Thus $y \sim_{f} x$.

Transitive: Let $x, y, z$ be such that $x \sim_{f} y$ and $y \sim_{f} z$. Then $f(x)=f(y)$ and $f(y)=f(z)$, so $f(x)=f(z)$. Thus $x \sim_{f} z$.

Therefore, $\sim_{f}$ is an equivalence relation on $A$. Finally, let $x, y, u, v \in A$ be such that $x \sim_{f} u$ and $y \sim_{f} v$. Then $f(x)=f(u)$ and $f(y)=f(v)$. Since $f$ is a UP-homomorphism, we get

$$
f(x \cdot y)=f(x) \bullet f(y)=f(u) \bullet f(v)=f(u \cdot v) .
$$

Thus $x \cdot y \sim_{f} u \cdot v$. By Lemma 1.9, we have $\sim_{f}$ is a congruence on $A$.

Theorem 2.3. Let $\left(A, \cdot, 0_{A}\right)$ and $\left(B, \bullet, 0_{B}\right)$ be UP-algebras, and $f: A \rightarrow B$ a UP-homomorphism. Then $\left(A / \sim_{f}, *_{,}\left(0_{A}\right) \sim_{f}\right)$ is a UP-algebra under the $*$ multiplication defined by $(x)_{\sim_{f}} *(y)_{\sim_{f}}=(x \cdot y)_{\sim_{f}}$ for all $x, y \in A$, called the quotient UP-algebra of $A$ induced by the congruence $\sim f$.

Proof. Let $x, y, u, v \in A$ be such that $(x)_{\sim_{f}}=(y)_{\sim_{f}}$ and $(u)_{\sim_{f}}=(v)_{\sim_{f}}$. Since $\sim_{f}$ is an equivalence relation on $A$, we get $x \sim_{f} y$ and $u \sim_{f} v$. By Lemma 1.9, we have $x \cdot u \sim_{f} y \cdot v$. Hence, $(x)_{\sim_{f}} *(u)_{\sim_{f}}=(x \cdot u)_{\sim_{f}}=(y \cdot v)_{\sim_{f}}=(y)_{\sim_{f}} *(v)_{\sim_{f}}$, showing $*$ is well defined.

$(U P-1)$ : Let $x, y, z \in A$. By (UP-1), we have $\left((y)_{\sim_{f}} *(z)_{\sim_{f}}\right) *\left(\left((x)_{\sim_{f}} *(y)_{\sim_{f}}\right) *\left((x)_{\sim_{f}} *(z)_{\sim_{f}}\right)\right)=((y \cdot z) \cdot((x \cdot y) \cdot(x \cdot z)))_{\sim_{f}}=\left(0_{A}\right) \sim_{\sim_{f}}$.

$(U P-2)$ : Let $x \in A$. By $(\mathrm{UP}-2)$, we have $\left(0_{A}\right) \sim_{f} *(x)_{\sim_{f}}=\left(0_{A} \cdot x\right)_{\sim_{f}}=(x)_{\sim_{f}}$.

(UP-3): Let $x \in A$. By (UP-3), we have $(x)_{\sim_{f}} *\left(0_{A}\right)_{\sim_{f}}=\left(x \cdot 0_{A}\right) \sim_{\sim_{f}}=\left(0_{A}\right) \sim_{f}$.

$(U P-4)$ : Let $x, y \in A$ be such that $(x)_{\sim_{f}} *(y)_{\sim_{f}}=(y)_{\sim_{f}} *(x)_{\sim_{f}}=\left(0_{A}\right)_{\sim_{f}}$. Then $(x \cdot y)_{\sim_{f}}=(y \cdot x)_{\sim_{f}}=\left(0_{A}\right) \sim_{\sim_{f}}$, it follows that $f(x) \bullet f(y)=$ $f(x \cdot y)=f\left(0_{A}\right)=f(y \cdot x)=f(y) \bullet f(x)$. By Theorem $1.16(1)$, we have $f(x) \bullet f(y)=f(y) \bullet f(x)=0_{B}$. By (UP-4), we have $f(x)=f(y)$. Thus $x \sim_{f} y$, so $(x)_{\sim_{f}}=(y)_{\sim_{f}}$.

Hence, $\left(A / \sim_{f}, *,\left(0_{A}\right) \sim_{f}\right)$ is a UP-algebra.

Theorem 2.4. Let $\left(A, \cdot, 0_{A}\right)$ and $\left(B, \bullet, 0_{B}\right)$ be UP-algebras, and $f: A \rightarrow B$ a UP-homomorphism. Then the mapping $\pi_{f}: A \rightarrow A / \sim{ }_{f}$ defined by $\pi_{f}(x)=(x)_{\sim_{f}}$ for all $x \in A$ is a UP-epimorphism, called the natural projection from $A$ to $A / \sim_{f}$. 
Proof. Let $x, y \in A$ be such that $x=y$. Then $(x)_{\sim_{f}}=(y)_{\sim_{f}}$, so $\pi_{f}(x)=\pi_{f}(y)$. Thus $\pi_{f}$ is well defined. Note that by the definition of $\pi_{f}$, we have $\pi_{f}$ is surjective. Let $x, y \in A$. Then

$$
\pi_{f}(x \cdot y)=(x \cdot y)_{\sim_{f}}=(x)_{\sim_{f}} *(y)_{\sim_{f}}=\pi_{f}(x) * \pi_{f}(y) .
$$

Thus $\pi_{f}$ is a UP-homomorphism. So we conclude that $\pi_{f}$ is a UP-epimorphism.

Theorem 2.5. (Fundamental Theorem of UP-homomorphisms) Let $\left(A, \cdot, 0_{A}\right)$ and $\left(B, \bullet, 0_{B}\right)$ be UP-algebras, and $f: A \rightarrow B$ a UPhomomorphism. Then there exists uniquely a UP-homomorphism $\varphi$ from $A / \sim_{f}$ to B such that $f=\varphi \circ \pi_{f}$. Moreover,

(1) $\pi_{f}$ is a UP-epimorphism and $\varphi$ a UP-monomorphism, and

(2) $f$ is a UP-epimorphism if and only if $\varphi$ is a UP-isomorphism.

As $f$ makes the following diagram commute,

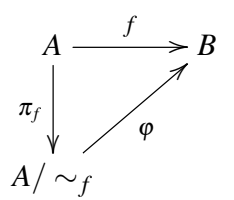

Proof. By Theorem 2.3, we have $\left(A / \sim_{f}, *,\left(0_{A}\right) \sim_{f}\right)$ is a UP-algebra. Define a mapping $\varphi: A / \sim_{f} \rightarrow B$ by

$$
\varphi\left((x)_{\sim_{f}}\right)=f(x) \text { for all }(x)_{\sim_{f}} \in A / \sim_{f} .
$$

Indeed, let $(x)_{\sim_{f}},(y)_{\sim_{f}} \in A / \sim_{f}$ be such that $(x)_{\sim_{f}}=(y)_{\sim_{f}}$. Then $x \sim_{f} y$, so

$$
\varphi\left((x)_{\sim_{f}}\right)=f(x)=f(y)=\varphi\left((y)_{\sim_{f}}\right) .
$$

For any $x, y \in A$, we see that

$$
\begin{aligned}
\varphi\left((x)_{\sim_{f}} *(y)_{\sim_{f}}\right) & =\varphi\left((x \cdot y)_{\sim_{f}}\right) \\
& =f(x \cdot y) \\
& =f(x) \bullet f(y) \\
& =\varphi\left((x)_{\sim_{f}}\right) \bullet \varphi\left((y)_{\sim_{f}}\right) .
\end{aligned}
$$

Thus $\varphi$ is a UP-homomorphism. Also, since

$$
\left(\varphi \circ \pi_{f}\right)(x)=\varphi\left(\pi_{f}(x)\right)=\varphi\left((x)_{\sim_{f}}\right)=f(x) \text { for all } x \in A,
$$

we obtain $f=\varphi \circ \pi_{f}$. We have shown the existence. Let $\varphi^{\prime}$ be a mapping from $A / \sim_{f}$ to $B$ such that $f=\varphi^{\prime} \circ \pi_{f}$. Then for any $(x) \sim_{f} \in A / \sim_{f}$, we have

$$
\begin{aligned}
\varphi^{\prime}\left((x)_{\sim_{f}}\right) & =\varphi^{\prime}\left(\pi_{f}(x)\right) \\
& =\left(\varphi^{\prime} \circ \pi_{f}\right)(x) \\
& =f(x) \\
& =\left(\varphi \circ \pi_{f}\right)(x) \\
& =\varphi\left(\pi_{f}(x)\right) \\
& =\varphi\left((x)_{\sim_{f}}\right) .
\end{aligned}
$$

Hence, $\varphi=\varphi^{\prime}$, showing the uniqueness.

(1) By Theorem 2.4, we have $\pi_{f}$ is a UP-epimorphism. Also, let $(x)_{\sim_{f}},(y)_{\sim_{f}} \in A / \sim_{f}$ be such that $\varphi\left((x)_{\sim_{f}}\right)=\varphi\left((y) \sim_{f}\right)$. Then $f(x)=f(y)$, so $x \sim_{f} y$. Thus $(x)_{\sim_{f}}=(y)_{\sim_{f}}$. Therefore, $\varphi$ a UP-monomorphism.

(2) Assume that $f$ is a UP-epimorphism. By (1), it suffices to prove $\varphi$ is surjective. Let $y \in B$. Then there exists $x \in A$ such that $f(x)=y$. Thus $y=f(x)=\varphi\left((x)_{\sim_{f}}\right)$, so $\varphi$ is surjective. Hence, $\varphi$ is a UP-isomorphism.

Conversely, assume that $\varphi$ is a UP-isomorphism. Then $\varphi$ is surjective. Let $y \in B$. Then there exists $(x)_{\sim_{f}} \in A / \sim_{f}$ such that $\varphi\left((x) \sim_{f}\right)=y$. Thus $f(x)=\varphi\left((x)_{\sim_{f}}\right)=y$, so $f$ is surjective. Hence, $f$ is a UP-epimorphism.

Theorem 2.6. (First UP-isomorphism Theorem) Let $\left(A, \cdot, 0_{A}\right)$ and $\left(B, \bullet, 0_{B}\right)$ be UP-algebras, and $f: A \rightarrow B$ a UP-homomorphism. Then

$$
A / \sim f \cong \operatorname{Im}(f) .
$$

Proof. By Theorem 1.16 (3), we have $\operatorname{Im}(f)$ is a UP-subalgebra of $B$. Thus $f: A \rightarrow \operatorname{Im}(f)$ is a UP-epimorphism. Applying Theorem 2.5 (2), we obtain $A / \sim f \cong \operatorname{Im}(f)$.

Lemma 2.7. Let $\left(A, \cdot, 0_{A}\right)$ and $\left(B, \bullet, 0_{B}\right)$ be UP-algebras, $f: A \rightarrow B$ a UP-homomorphism, and $H$ a UP-subalgebra of A. Denote $H_{\sim_{f}}=\bigcup_{h \in H}(h)_{\sim_{f}}$. Then $H_{\sim_{f}}$ is a UP-subalgebra of $A$. 
Proof. Clearly, $\emptyset \neq H_{\sim_{f}} \subseteq A$. Let $a, b \in H_{\sim_{f}}$. Then $a \in(x)_{\sim_{f}}$ and $b \in(y)_{\sim_{f}}$ for some $x, y \in H$, so $(a)_{\sim_{f}}=(x)_{\sim_{f}}$ and $(b)_{\sim_{f}}=(y)_{\sim_{f}}$. Theorem 2.3 gives $\left(A / \sim_{f}, *,\left(0_{A}\right) \sim_{f}\right)$ is a UP-algebra, so

$$
(a \cdot b)_{\sim_{f}}=(a)_{\sim_{f}} *(b)_{\sim_{f}}=(x)_{\sim_{f}} *(y)_{\sim_{f}}=(x \cdot y)_{\sim_{f}} .
$$

Thus $a \cdot b \in(x \cdot y)_{\sim_{f}}$. Since $x, y \in H$, it follows from Proposition 1.7 that $x \cdot y \in H$. Thus $a \cdot b \in(x \cdot y)_{\sim_{f}} \subseteq H_{\sim_{f}}$. Hence, $H_{\sim_{f}}$ is a UP-subalgebra of $A$.

Theorem 2.8. (Second UP-isomorphism Theorem) Let $\left(A, \cdot, 0_{A}\right)$ and $\left(B, \bullet, 0_{B}\right)$ be UP-algebras, $f: A \rightarrow B$ a UP-homomorphism, and $H$ a UP-subalgebra of A. Denote $H_{\sim_{f}} / \sim_{f}=\left\{(x)_{\sim_{f}} \mid x \in H_{\sim_{f}}\right\}$. Then

$$
H / \sim_{\left.\pi_{f}\right|_{H}} \cong H_{\sim_{f}} / \sim_{f} .
$$

Proof. By Lemma 2.7, we have $H_{\sim_{f}}$ is a UP-subalgebra of $A$. Then it is easy to check that $H_{\sim_{f}} / \sim_{f}$ is a UP-subalgebra of $A / \sim_{f}$, thus $\left(H_{\sim_{f}} / \sim_{f}, *,\left(0_{A}\right)_{\sim_{f}}\right)$ itself is a UP-algebra. Also, it is obvious that $H \subseteq H_{\sim_{f}}$, then

$$
\left(\left.\pi_{f}\right|_{H}=\right) g: H \rightarrow H_{\sim_{f}} / \sim_{f}, x \mapsto(x)_{\sim_{f}},
$$

is a mapping. Indeed, $g$ is the restriction of $\pi_{f}$ to $H$. Thus $g$ is a UP-epimorphism. Indeed, $H_{\sim_{f}} / \sim_{f}=H / \sim_{f}$. Theorem 2.6 gives $H / \sim_{\left.\pi_{f}\right|_{H}} \cong H_{\sim_{f}} / \sim_{f}$.

Theorem 2.9. Let $\left(A, \cdot, 0_{A}\right)$ and $\left(B, \bullet, 0_{B}\right)$ be UP-algebras, $f: A \rightarrow B$ and $g: A \rightarrow B$ UP-homomorphisms with $\sim_{f} \subseteq \sim_{g}$. Define the binary relation $\sim_{g} / \sim_{f}$ on $A / \sim_{f}$ as follows: for all $x, y \in A$,

$$
(x)_{\sim_{f}} \sim_{g} / \sim_{f}(y)_{\sim_{f}} \text { if and only if } x \sim_{g} y .
$$

Then $\sim_{g} / \sim_{f}$ is a congruence on $A / \sim_{f}$.

Proof. By Theorem 2.3, we have $\left(A / \sim_{f}, *,\left(0_{A}\right)_{\sim_{f}}\right)$ is a UP-algebra.

Reflexive: For all $x \in A$, we have $x \sim_{g} x$. Thus $(x) \sim_{f} \sim_{g} / \sim_{f}(x)_{\sim_{f}}$.

Symmetric: Let $x, y \in A$ be such that $(x) \sim_{f} \sim_{g} / \sim_{f}(y) \sim_{f}$. Then $x \sim_{g} y$, so $y \sim_{g} x$. Thus $(y) \sim_{f} \sim_{g} / \sim_{f}(x) \sim_{f}$.

Transitive: Let $x, y$, ze such that $(x)_{\sim_{f}} \sim_{g} / \sim_{f}(y)_{\sim_{f}}$ and $(y)_{\sim_{f}} \sim_{g} / \sim_{f}(z) \sim_{f}$. Then $x \sim_{g} y$ and $y \sim_{g} z$, so $x \sim_{g} z$. Thus $(x) \sim_{f} \sim_{g} / \sim_{f}(z) \sim_{f}$. Therefore, $\sim_{g} / \sim_{f}$ is an equivalence relation on $A / \sim_{f}$. Finally, let $x, y, u, v \in A$ be such that $(x)_{\sim_{f}} \sim_{g} / \sim_{f}(u) \sim_{f}$ and $(y) \sim_{f} \sim_{g} / \sim_{f}(v) \sim_{f}$. Then $x \sim_{g} u$ and $y \sim_{g} v$. The binary relation $\sim_{g}$ is a congruence on $A$ by Theorem 2.2, that is $x \cdot y \sim_{g} u \cdot v$. Thus $(x \cdot y) \sim_{f} \sim_{g} / \sim_{f}(u \cdot v) \sim_{f}$, so $(x)_{\sim_{f}} *(y)_{\sim_{f}} \sim_{g} / \sim_{f}(u)_{\sim_{f}} *(v)_{\sim_{f}}$. Hence, $\sim_{g} / \sim_{f}$ is a congruence on $A / \sim_{f}$.

Theorem 2.10. (Third UP-isomorphism Theorem) Let $\left(A, \cdot, 0_{A}\right)$ and $\left(B, \bullet, 0_{B}\right)$ be UP-algebras, $f: A \rightarrow B$ and $g: A \rightarrow B$ UP-homomorphisms with $\sim_{f} \subseteq \sim_{g}$. Then

$$
\left(A / \sim_{f}\right) /\left(\sim_{g} / \sim_{f}\right) \cong A / \sim_{g}
$$

Proof. By Theorem 2.3, we obtain $\left(A / \sim_{f}, *,\left(0_{A}\right) \sim_{f}\right)$ and $\left(A / \sim_{g}, *^{\prime},\left(0_{A}\right) \sim_{g}\right)$ are UP-algebras. By Theorem 2.4, we obtain

$$
\pi_{f}: A \rightarrow A / \sim_{f}, x \mapsto(x)_{\sim_{f}}
$$

and

$$
\pi_{g}: A \rightarrow A / \sim_{g}, x \mapsto(x)_{\sim_{g}}
$$

are UP-epimorphisms. Applying Theorem 2.5 (2), there exists a UP-isomorphism

$$
g / f: A / \sim_{f} \rightarrow A / \sim_{g},(x)_{\sim_{f}} \mapsto(x)_{\sim_{g}} .
$$

Indeed, $A / \sim_{f} \cong A / \sim_{g}$. By Theorem 2.9 and 2.3, we have $\left(A / \sim_{f}\right) / \sim_{g / f}$ is a UP-algebra. By Theorem 2.4, we obtain

$$
\pi_{g / f}: A / \sim_{f} \rightarrow\left(A / \sim_{f}\right) / \sim_{g / f},(x)_{\sim_{f}} \mapsto\left((x) \sim_{f}\right) \sim_{g / f}
$$

is a UP-epimorphism. Applying Theorem 2.5 (2), there exists a UP-isomorphism

$$
\varphi:\left(A / \sim_{f}\right) / \sim_{g / f} \rightarrow A / \sim_{g},\left((x)_{\sim_{f}}\right) \sim_{g / f} \mapsto(x)_{\sim_{g}} .
$$

That is,

$$
\left(A / \sim_{f}\right) / \sim_{g / f} \cong A / \sim_{g} .
$$

We shall show that $\sim_{g / f}=\sim_{g} / \sim_{f}$. For any $(x)_{\sim_{f}},(y)_{\sim_{f}} \in A / \sim_{f}$,

$$
\begin{aligned}
(x)_{\sim_{f}} \sim_{g / f}(y)_{\sim_{f}} & \Leftrightarrow(g / f)\left((x)_{\sim_{f}}\right)=(g / f)\left((y)_{\sim_{f}}\right) \\
& \Leftrightarrow(x)_{\sim_{g}}=(y)_{\sim_{g}} \\
& \Leftrightarrow x \sim_{g} y \\
& \Leftrightarrow(x)_{\sim_{f}} \sim_{g} / \sim_{f}(y)_{\sim_{f}}
\end{aligned}
$$

by (2.1) and (2.4). Thus $\sim_{g / f}=\sim_{g} / \sim_{f}$. Hence, $\left(A / \sim_{f}\right) /\left(\sim_{g} / \sim_{f}\right) \cong A / \sim_{g}$. 
Corollary 2.11. Let $\left(A, \cdot, 0_{A}\right)$ and $\left(B, \bullet, 0_{B}\right)$ be UP-algebras, $f: A \rightarrow B$ a UP-homomorphism, and $C$ a UP-ideal of $A$. Then

$$
A / \sim_{C} \cong A / \sim_{f}
$$

As $\pi_{f}$ makes the following diagram commute,

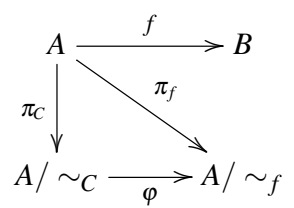

Proof. It is straightforward by Theorem 1.12, 1.14, 2.4, and 2.5 (2).

\section{Acknowledgment}

The authors wish to express their sincere thanks to the referees for the valuable suggestions which lead to an improvement of this paper.

\section{References}

[1] S. Asawasamrit, KK-isomorphism and its properties, Int. J. Pure Appl. Math. 78 (2012), no. 1, 65-73.

[2] K. H. Dar and M. Akram, On K-homomorphisms of K-algebras, Int. Math. Forum 2 (2007), no. 46, $2283-2293$.

[3] J. Hao and C. X. Li, On ideals of an ideal in a BCI-algebra, Sci. Math. Jpn. (in Editione Electronica) 10 (2004), no. 16, 493-500.

[4] Q. P. Hu and X. Li, On BCH-algebras, Math. Semin. Notes, Kobe Univ. 11 (1983), 313-320.

[5] A. Iampan, The UP-isomorphism theorems for UP-algebras, Manuscript submitted for publication, April 2015.

[6] A. Iampan, A new branch of the logical algebra: UP-algebras, J. Algebra Relat. Top. 5 (2017), no. 1, 35-54.

[7] Y. Imai and K. Iséki, On axiom system of propositional calculi, XIV, Proc. Japan Acad. 42 (1966), no. 1, 19-22.

[8] K. Iséki, An algebra related with a propositional calculus, Proc. Japan Acad. 42 (1966), no. 1, $26-29$.

[9] Y. B. Jun, S. M. Hong, X. L. Xin, and E. H. Roh, Chinese remainder theorems in BCI-algebras, Soochow J. Math. 24 (1998), no. 3, $219-230$.

[10] S. Keawrahun and U. Leerawat, On isomorphisms of SU-algebras, Sci. Magna 7 (2011), no. 2, 39-44.

[11] C. B. Kim and H. S. Kim, On BG-algebras, Demonstr. Math. 41 (2008), no. 3, 497-505.

[12] K. H. Kim, On structure of KS-semigroup, Int. Math. Forum 1 (2006), no. 2, 67-76.

[13] J. S. Paradero-Vilela and M. Cawi, On KS-semigroup homomorphism, Int. Math. Forum 4 (2009), no. 23, 1129-1138.

[14] J. K. Park, W. H. Shim, and E. H. Roh, On isomorphism theorems in IS-algebras, Soochow J. Math. 27 (2001), no. $2,153-160$.

[15] C. Prabpayak and U. Leerawat, On ideals and congruences in KU-algebras, Sci. Magna 5 (2009), no. 1, 54-57. 